\title{
Aplicación del CAPM en Mercados Emergentes: Una revisión teórica
}

\section{CAPM Application in Emerging Markets: A theoretical review}

Juan Calos Ruiz Barrezueta ${ }^{1}$, Jorge Enrique Altamirano Flores ${ }^{2}$, Luis Bernardo Tonon Ordóñez ${ }^{3}$

INFORMACIÓN DEL

\section{ARTÍCULO}

Fecha de recepción: 12 de Marzo de 2021.

Fecha de aceptación: 28 de Mayo de 2021.

${ }^{1}$ Master of Science in Accounting and Corporate Finance, Oklahoma City University. Docente-investigador,

Universidad Internacional del Ecuador. E-mail: juruizba@uide.edu.ec

Código ORCID:

https://orcid.org/0000-0003-2354-1638

$2 \mathrm{PhD}$ Development Economics, Newcastle University. Docenteinvestigador, Universidad Internacional del Ecuador

E-mail: joaltamiranofl@uide.edu.ec

Código ORCID:

https://orcid.org/0000-0003-3882-2432

3 Maestría en Administración de Empresas, Universidad del Azuay. Docente-investigador, Universidad del Azuay - Ecuador.

E-mail: 1tonon@uazuay.edu.ec

Código ORCID:

https://orcid.org/0000-0003-2360-9911

CITACIÓN: Ruíz Barrezueta, J.C., Altamirano Flores, J.E., \& Tonon Ordóñez, L.B. (2021). Aplicación del CAPM en Mercados Emergentes: Una revisión teórica. Podium, 39, 53-70. doi:10.31095/podium.2021.39.4

\section{ENLACE DOI:}

http://dx.doi.org/10.31095/podium.202 1.39 .4

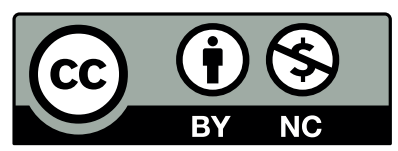

\section{Resumen}

El objetivo de este artículo es investigar teóricamente la aplicabilidad del modelo de precios de activos de capital en mercados emergentes. El modelo mide el riesgo utilizando el coeficiente beta, que se deriva de un equilibrio, mismo que el modelo asume como constante. Sin embargo, el uso del modelo CAPM en mercados emergentes resulta complejo e incluso controversial. Mediante una revisión teórica y sistemática, este estudio recoge las contribuciones publicadas desde el año 1964 hasta el año 2021 en las bases de datos Scopus, JSTOR, red Redalyc y el sistema Dialnet. Se exploró analítica y cronológicamente las contribuciones destacando la literatura a favor y en contra del modelo, lo que permitió concluir que existen varias fórmulas con diferentes variables propuestas por autores que han tratado de acomodar el modelo a las condiciones que presentan los mercados emergentes, sin embargo, queda claro que no existe una fórmula universal.

\section{Palabras Clave:}

CAPM, Mercados emergentes, Beta, Riesgo país, Riesgo Sistemático, Valoración de Activos.

Clasificación JEL: G12.

\begin{abstract}
The objective of this paper is to theoretically investigate the applicability of the capital asset pricing model in emerging markets. The model measures risk by beta, which follows from an equilibrium, in which the CAPM model assumes that equilibrium conditions do not change. However, the use of the CAPM model in emerging markets has proved challenging and even controversial. Using a theoretical and systematic review, this study acknowledges contributions published from 1964 to 2021 in databases such as Scopus, JSTOR, Redalyc and Dialnet. In addition, this study analytically and chronologically explores those contributions highlighting the literature in favor of the model and the literature against the model, which allowed to conclude that there are several formulas with different variables proposed by authors who have tried to accommodate the model to the conditions of emerging markets, however, it is clear that there is no a universal formula.
\end{abstract}

\section{Keywords:}

CAPM, Emerging Markets, Risk-Free Rate, Beta, Country Risk, Systematic Risk, Asset Valuation.

JEL Classification: G12.

\section{3}

PODIUM No. 39, Junio 2021, pp. 53-70

(C) Universidad Espíritu Santo - UEES

ISSN: 1390-5473 e-ISSN: 2588-0969 


\section{Introducción}

En un mercado en equilibrio, que usualmente se encuentra en países desarrollados, las personas que asumen riesgos suelen ser recompensadas vía los rendimientos obtenidos (Campos, Castro, Cuy, y Ferrer, 2005). Esta es la base con la que funciona el CAPM, es decir, el rendimiento está intrínsecamente vinculado al riesgo (Wong y Chirinos, 2016). El modelo CAPM funciona bajo el supuesto de que en equilibrio el riesgo específico de las empresas no es valorado (Butt y Sadaqat, 2020). Sin embargo, el supuesto de un mercado en equilibrio, no se aplica de la misma manera en países en vías de desarrollo, en donde los mercados son considerados como emergentes. La literatura ha producido una gran cantidad de evidencia inconclusa sobre un modelo de valoración que sea adecuado para mercados emergentes (Bai y Green, 2020). La distinción entre mercados desarrollados y mercados emergentes cobró importancia durante la década de los 80s (Bekaert \& Harvey, 2013) cuando el mundo experimentó una ola generalizada de privatizaciones y los compradores, vendedores e intermediarios financieros notaron que era necesario un marco referencial para valorar activos en las diferentes regiones del mundo (Abuaf, 2011). Desde entonces se ha producido una considerable cantidad de información académica sobre los mercados emergentes (Cavusgil, 2021). Las empresas tradicionalmente han tratado de crecer mediante la adquisición de otras empresas en países desarrollados (Obstfeld, Ostry, y Qureshi, 2019). Sin embargo, en la actualidad las inversiones en mercados emergentes han cobrado mayor importancia por lo que la necesidad de valorarlas es un imperativo (Britzelmaier, 2009).

Investigaciones relacionadas a las finanzas corporativas evidencian una tendencia hacia el uso de una tasa de descuento "apropiada" para el costo de capital basada en la teoría del CAPM (Pereiro, 2006). Los analistas suelen utilizar un amplio espectro de modelos para valorar los activos financieros mediante el cálculo de una tasa de descuento. Estos modelos pueden ser muy sencillos o muy sofisticados (Damodaran, 2007). La tasa de descuento es el costo de capital que se utiliza para valorar instrumentos financieros, proyectos o empresas. Determinar adecuadamente esa tasa de descuento es de suma importancia, debido a que de esta manera se puede evitar la sobrevaluación o subvaluación de proyectos.

La literatura sobre valoración de proyectos o empresas, se basa en la información e indicadores de mercados desarrollados como el de la Eurozona o el de Estados Unidos; o en su defecto; en mercados emergentes, pero muy desarrollados, como el de la India, Brasil, Argentina o México (Sabal 2008). Las sistemáticas de valoración empleadas en los países antes mencionados omiten el hecho de que factores como el nivel de ingresos, riesgo de liquidez, apalancamiento, apertura a la inversión extranjera, modelos político-económicos, riesgo país, riesgo de pérdidas, volatilidad, tipos de cambio, entre otros, 
(Álvarez, Ortega, Sánchez, y Herrera, 2004) distan mucho de la realidad de mercados emergentes de países como Ecuador, Bolivia, Colombia y Perú, cuyas realidades son muy diferentes a la de los mercados desarrollados (Bruner, Conroy, Estrada, Kritzman, y Li, 2002).

Los mercados emergentes, como la India, Brasil y México, si bien siguen teniendo rentabilidades por encima de mercados desarrollados como los de España, Estados Unidos y Japón, según datos de la Conferencia de las Naciones Unidas sobre Comercio y Desarrollo (UNCTAD, 2020), éstos son cada vez más eficientes, eliminando asimetrías de información, y reduciendo las ganancias extraordinarias en dichos mercados. Es así, que otras economías de países como Colombia, Perú, Ecuador y Bolivia, se convierten en excelentes opciones de inversión. De esto último, cabe recalcar que los efectos de la pandemia siguen teniendo incidencia directa en lo que se refiere a Inversión Extranjera Directa (IED), donde los flujos han experimentado serias reducciones en todas las naciones independientemente de su economía. De acuerdo con Bruner et. al. (2002), la brecha existente entre los mercados desarrollados y los emergentes al momento de valorar activos financieros, sigue siendo muy amplia, y se evidencia aún más, si se considera la información que se encuentra disponible en los mercados emergentes.

El presente estudio tiene por objetivo analizar la aplicación del CAPM en mercados emergentes, especialmente aquellos cuyo nivel de desarrollo se encuentra en etapas relativamente tempranas. La metodología utilizada para la investigación se basa en una revisión descriptiva y analítica.

\section{Revisión de literatura}

\section{Antecedentes}

La valoración es el centro de la teoría financiera (García-Sánchez, Preve, y Sarria-Allende, 2010) y en la literatura se puede evidenciar que existen varias metodologías para valorar activos financieros. El modelo CAPM es sin duda una de las teorías más frecuentemente utilizada, a pesar de que su utilización ha sido controversial desde su misma introducción a inicios de los años 60s (Elbannan, 2015). Desde un punto de vista cronológico se debería iniciar citando "La Teoría de Portafolio o Modelo de Medias Varianzas" de Markowitz (1952). Esta teoría se refiere a la relación que existe entre el riesgo y rendimiento, pero siendo su gran aporte al área de las finanzas, la investigación acerca de la combinación que existe al juntar varios instrumentos financieros en una cartera, y así optimizar el rendimiento y el riesgo respectivo. $\mathrm{La}$ Teoría de Markowitz se basa en que los inversores por naturaleza tienen aversión al riesgo, por lo que se asume en el caso de existir dos activos financieros con igual rentabilidad, que los inversionistas optarían por el activo con menor exposición al riesgo, siendo claro que, al incrementarse el riesgo, cualquier inversionista debería exigir mayor compensación o rentabilidad.

Markowitz (1952) definió en su 
teoría, el hecho de que el inversionista basa sus decisiones de acuerdo a la conformación óptima de su portafolio, es decir, diversificando mediante el uso de indicadores como la media o rendimiento aritmético como medida de rentabilidad y la varianza o desviación estándar como medidas de riesgos. La utilidad esperada está determinada en función de la relación existente entre el riesgo y la rentabilidad, tomando en consideración las ponderaciones de los distintos instrumentos financieros que conforman el portafolio, y la correlación existente entre sí, optimizando el principio de diversificación. El portafolio que puede llegar a conformar el inversionista podría tener una infinidad de combinaciones y ponderaciones de los activos financieros, pero se podría facilitar la toma de decisiones mediante el modelo de eficiencia en donde se compense el riesgo con cada nivel de retorno esperado. Por lo expuesto, se puede concluir que el modelo de Markowitz no pretende maximizar el rendimiento o minimizar el riesgo, lo que plantea es enfocarse en un conjunto de portafolios eficientes y óptimos, más que en una solución única.

Sharpe (1964) introdujo el "Modelo de Mercado", que no es contradictorio con la Teoría de Portafolio de Markowitz, por el contrario, es derivado del mismo, introduciendo dos hipótesis:

1) La relación entre dos activos financieros, se debe a su común relación con la cartera del mercado.

2) La relación de cada instrumento financiero de la cartera y el mercado es lineal.

Tal como Markowitz, Sharpe (1694) señala la relación directa que existe entre el riesgo y el retorno esperado de una inversión, pero tomando en cuenta los riesgos sistemáticos y no sistemáticos, siendo la suma de estos dos, el riesgo total del portafolio. Un portafolio bien diversificado, contemplaría expresamente el riesgo sistemático.

\section{Modelo de Valoración de Activos Financieros CAPM}

Sharpe (1964) desarrolló el "Modelo de Valoración de Activos Financieros CAPM" (Capital Asset Pricing Management) que se muestra en la ecuación 1:

$$
\mathrm{Ri}=\mathrm{Rf}+\operatorname{Beta} *(\mathrm{Rm}-\mathrm{Rf})
$$

\section{Donde:}

Ri es la tasa mínima esperada.

Rf es la tasa Libre de Riesgo.

Beta es el indicador de Riesgo del Activo.

Rm es el Rendimiento del Mercado el cual sirve para calcular la rentabilidad del activo, en función de su riesgo.

El modelo CAPM es, sin duda, el que ha tenido mayor aceptación hasta el día de hoy, debido a que ofrece una predicción poderosa e intuitiva de cómo medir el riesgo y la relación entre la tasa de retorno esperada y el riesgo (Fama y French, 2004). Sharpe (1964) introduce para este modelo un indicador de la volatilidad de un activo con relación al mercado llamado "beta", el mismo que mide la sensibilidad de la rentabilidad del activo, frente a cambios de rentabilidad suscitados en el mercado. 
El modelo CAPM, por el efecto de la diversificación, no presenta riesgo no sistemático, solo sistemático, es decir, se plantea una relación creciente entre beta, rendimiento y riesgo. En otras palabras, a mayor riesgo, mayor será la compensación que exijan los inversionistas. El modelo de valoración de activos CAPM, sirve para valorar tanto instrumentos financieros como para valorar proyectos. El modelo CAPM asume, que el mercado es perfectamente competitivo donde los inversores podrían optimizar sus utilidades dependiendo del grado de dispersión que existe entre el rendimiento del activo y la respectiva relación con el mercado. Se concluye, que en teoría, no existe asimetría de información, pues todos los inversores tienen acceso a la misma, la cual es actualizada y en tiempo exacto.

\section{Aportes al modelo}

Durante la década de los 70s, se desarrolló el "Modelo CAPM Multifactor" el cual considera que la incertidumbre acerca del precio futuro de un activo no debería ser el único riesgo que se debería tomar en cuenta, sino que existen otros riesgos que afectan el poder adquisitivo o recursos del inversionista (Merton, 1973). Los riesgos que comprometen las variables mencionadas son: 1) Riesgos de Inestabilidad de Recursos, 2) Riesgo de Inestabilidad de Precios Futuros, 3) Riesgo de Oportunidades Futuras. Este modelo se deriva del original CAPM de Sharpe, incorpora nuevos riesgos que afectan a la inversión y que son ajenos al mercado. Es por ello, que este modelo se enfoca en la prima de riesgo, y manifiesta que los inversionistas deberían ser recompensados por cada riesgo adicional asumido además del mercado. Por supuesto, de no existir ningún riesgo adicional al mercado, este modelo simplificará al modelo CAPM original.

Ross (1976) desarrolló el "Modelo de la Teoría por Valoración de Arbitraje o APT" (Arbitraje Pricing Theory) que tiene como objetivo, estimar la prima de riesgo, es decir el riesgo adicional entre la tasa libre de riesgo y el rendimiento del mercado. El APT, en contraste con el modelo CAPM, considera la posibilidad que existan diversos riesgos sistemáticos, y su rentabilidad condicionada por factores de carácter anticipables y no anticipables. Los primeros factores estarían reflejados en el precio, en caso de que el activo se encontrase en un mercado eficiente, y los segundos, donde el inversor podría conocer los riesgos sistemáticos existentes y la sensibilidad de los activos a la exposición de dichos factores.

En el modelo APT, los inversionistas esperarían ser compensados por el riesgo sistemático asumido, mismo que afectaría el rendimiento del activo. Dicha compensación puede derivarse de la diferencia entre la tasa libre de riesgo y el rendimiento del activo, y la valoración del riesgo sistemático medido por el indicador beta. De acuerdo con Ross (1976), a diferencia de los modelos CAPM original y multifactor, el modelo APT es menos restrictivo o más flexible en cuanto a la preferencia de los inversionistas con relación al riesgo y al rendimiento. 
Breeden (1979) desarrolla el consumption CAPM conocido también como el C-CAPM en el cual se mide el rendimiento de una cartera por medio del precios de los activos de consumo y el consumo generado en un mercado en un período dado de tiempo. La propuesta incorpora una medida de riesgo denominada Beta de consumo que toma en consideración el crecimiento del consumo como una forma de tener en cuenta implícitamente la riqueza de los consumidores-inversionistas. El autor reconoce en sus conclusiones ventajas e inconvenientes de su propuesta destacándose en las últimas que los datos reales de consumo que están disponibles contienen un error de medición considerable, mientras que los precios y la cantidad de acciones utilizados en los cálculos de la cartera de mercado se miden con muy poco error.

Otro aporte al modelo es realizado por Estrada (2002) quien propone el Downside CAPM o D-CAPM que es similar al CAPM pero que toma en cuenta que los inversionistas tienden a fijarse en los rendimientos menores al rendimiento medio o, en otras palabras, más en la parte negativa del riesgo. Para la medición del riesgo se crea un nuevo indicador alternativo denominado downside beta o beta a la baja que se calcula en base a la semivarianza de los rendimientos con respecto a la media. Por el supuesto de que las rentabilidades no presentan una distribución normal, como lo hace el CAPM, este modelo es aplicable a mercados emergentes. Estrada concluye que su modelo explica casi el 55\% de la variabilidad en la sección transversal de los retornos en los mercados emergentes. Así como también que los rendimientos en los mercados emergentes son mucho más sensibles a las diferencias en el beta a la baja que a las diferencias iguales en el beta tradicional. Además, de que el D-CAPM genera retornos promedio requeridos sobre el capital de más de $2.5 \%$ al año superiores a los generados por el CAPM.

\section{Críticas al modelo}

Fama y French (1996) discrepan sobre el ajuste empírico del modelo CAPM de Sharpe (1964) ya que indican que al modificar el beta del mercado, siendo este no acorde al tamaño respectivo, la relación univariante entre el beta y el rendimiento promedio, sería muy débil, además que el indicador beta no es suficiente para determinar el rendimiento promedio.

A pesar de las críticas, Sharpe (1990) afirma que debido a los avances tecnológicos de las últimas décadas, la creciente integración de los mercados, y el mejor entendimiento de la economía financiera, los mercados de capitales comienzan progresivamente asemejarse a los escenarios simples que sugería el modelo CAPM, por lo que su empleabilidad no pierde vigencia. Debido a que el modelo CAPM asume que todos los inversionistas son adversos al riesgo, el riesgo y rendimiento serían aceptados, solo si éstos estuviesen por encima de la base de retorno esperado.

\section{CAPM en mercados emergentes y el riesgo país}

Es evidente que el modelo CAPM, 
icono de la valoración de activos financieros, tiene factores y suposiciones técnicas al momento de la valoración, que en la práctica, no son acordes con los mercados emergentes. Mariscal y Lee (1993) propusieron la adición relativa de un indicador llamado riesgo país como medida de ajuste al modelo CAPM en mercados emergentes. El riesgo país es un indicador financiero y económico que señala la probabilidad de un país en incumplir sus obligaciones financieras y crediticias internacionales (Harvey, 2005). Este indicador hace referencia a todo riesgo concerniente tanto de carácter crediticio, político, de estabilidad gubernamental, así como de inversión, por lo que entre más alto sea el mismo, mayor será la percepción o grado de incumplimiento por parte del mercado acerca del país en cuestión, por lo que será más alto su costo de inversión o de deuda (Kiguel y Lopetegui, 1997; Lee y Wang, 2020). Este factor de riesgo, tomaría en cuenta la diferencia entre la rentabilidad de un bono de Estados Unidos y la rentabilidad de un bono soberano del país en estudio, y así, compensar la exposición al riesgo adicional que inversionistas en países con mercados desarrollados asumen al invertir en mercados emergentes (Timurlenk y Kaptan, 2012). Pero hay que tomar en cuenta que como afirman Botero y Vecino el riesgo país "es subjetivo y cae en errores conceptuales que terminan deslegitimándolo" (2015, p 39).

El riesgo país debería sumarse al resultado obtenido al calcular la tasa de descuento utilizando el modelo CAPM, para que de esta manera se pueda ajustar en cierto grado a la realidad del país en donde se piensa realizar la inversión. Contrastando con esta inclusión Godfrey y Espinosa (1996) afirman que el adicionar al CAPM el riesgo país lleva a un error de duplicación pues parte de la prima por riesgo país puede estar ya incluida en la prima por riesgo de mercado. En concordancia con lo anterior, Sabal (2002) propone que el riesgo país no debería ser ajustado directamente al modelo CAPM, ya que esto implicaría que esta medida de riesgo, es sistemática, es decir, no diversificable. Además, que, en muchos casos, este riesgo país es incluido de igual manera indirectamente en los flujos de caja para mitigar las incertidumbres no solo del proyecto, sino de la industria y del país en estudio (Sabal, 2008).

Añadir el indicador de riesgo país sería un error, ya que no todos los proyectos o activos son afectados de la misma manera por este indicador (Sabal, 2004). Los mercados emergentes tienen cierta inestabilidad política adicional a economías de países desarrollados, por lo que ciertos sectores se ven mayormente afectados que otros. Por ejemplo, existen ciertas industrias que tienen mayor afectación en la población en general y podrían requerir intervención gubernamental como son las empresas públicas de servicios básicos (Kiguel \& Lopetegui, 1997). Otras industrias, como las pertenecientes a los negocios de alimentación como los restaurantes, no son tan sensibles a efectos sociales o no dependen de manera directa de la política pública (Damodaran, 2003). 
Sabal (2002) se opone a incluir directamente el riesgo país al modelo CAPM, debido que al calcular la prima de riesgo, usando los bonos soberanos del país en estudio, como la tasa libre de riesgo, especialmente de países en vías de desarrollo; el riesgo de incumplimiento o "default" podría ya estar implícitamente en el cálculo de esta brecha distorsionando la realidad del riesgo en dicha inversión. Debido a que el riesgo país tiene un impacto de naturaleza geométrico sobre el valor presente de los flujos del proyecto, es decir, que mientras más largo sea el horizonte de tiempo, mayor efecto tendrá este sobre el VPN (valor presente neto) del proyecto. A medida que transcurre el tiempo, los inversionistas podrían desarrollar medidas óptimas para mitigar los efectos del riesgo país.

La disyuntiva se encuentra en determinar si el riesgo país es realmente sistemático, este dilema es difícil de responder puesto que la definición de este indicador de riesgo puede tornarse subjetiva, dependiendo del inversionista (Timurlenk y Kaptan, 2012). Teóricamente este indicador se refiere solo a la probabilidad de incumplimiento, pero existen otros riesgos como grado de corrupción, inestabilidad jurídica, constitucional entre otros, que no están contemplados en el indicador (Kiguel y Lopetegui, 1997).

Otras variables a tomarse en cuenta en mercados emergentes

Sabal (2002), indica que otra manera de reflejar el riesgo y rendimiento de una inversión podría ser a través del índice de mercado valores en cada país, sin embargo este método sólo sería aplicable si se contase con un mercado desarrollado, con información verificable y actualizada constantemente (Kruschwitz, Löffler, y Mandl, 2012), en donde este índice pueda ser un reflejo de la economía del país como sucede en países como Estados Unidos, España, o en vías de desarrollo como Brasil, México o India. Por lo expuesto, se puede concluir que no es una tarea fácil poder discernir cuán diversificable es el riesgo país, por ende, lo inadecuado de simplemente sumar el mismo al modelo CAPM. Solnik (1974) propuso el "Modelo del CAPM Internacional Modificado" el cual se basa en que se debería utilizar un beta ponderado o ajustado de acuerdo a los flujos que genera en la operación del país en estudio y su relación con el índice del mercado respectivo. Se puede concluir y proponer, que el riesgo país debería ser integrado en los flujos del proyecto, para que así todas las variables y riesgos sean debidamente contempladas. Sabal (2002) propone un mecanismo de ponderación de los betas de las industrias que conforman los mercados en vías de desarrollo, en donde se incorpora indirectamente el riesgo país.

Damodaran (2002) sugiere que al riesgo país, se le debería ajustar la desviación de la prima de riesgo de un mercado accionario emergente, con la prima de riesgo de un mercado desarrollado, y de esta manera, combinando estos mecanismos, se podría consensuar la relación riesgo y rentabilidad en mercados de economías 
en vías de desarrollo. No obstante, el hecho que, no todos los proyectos o activos de un país pueden ser afectados por el riesgo país equitativamente, esto debido a que, cada industria o empresa tiene diferente exposición a este factor, así como diferente grado de liquidez (Sabal, 2002). Por esta razón se propone un factor llamado Lambda, que pondera este riesgo entre empresas o activos de una industria (Damodaran, 2003). A todo esto, Kruschwitz et al (2012) afirman que esta variante o ajuste al modelo, carece de fundamento teórico y empírico, puesto que no se establece una definición clara de lo que contempla el riesgo país.

Los críticos al modelo CAPM, Fama y French (2003) aducen que dicho modelo nunca podría ser exitoso empíricamente, puesto a que éste concluye que la relación entre el rendimiento promedio y el beta del mercado es más plano (la prima de riesgo por unidad de beta de mercado es menor) que los resultados predichos por el modelo, lo cual es suficiente para invalidar la aplicación del mismo. A pesar del desacuerdo por parte de varios autores, Perold (2004) realiza un análisis del modelo CAPM con sus extensiones, y describe las particularidades de dicho modelo. Por ejemplo, indica que todos los inversionistas deben ser adversos al riesgo, los mercados son perfectos, es decir que todos los activos son infinitamente divisibles, no existen costos de transacción, no existe asimetría de información, todos los inversionistas pueden acceder o prestar sus recursos a la tasa libre de riesgo y todos los inversionistas tienen acceso a las mismas oportunidades de inversión. Si bien, estas características solo ocurrirían en un escenario perfecto, se reconoce que este modelo podría ser una base para predecir el comportamiento futuro de los inversores, así como para comprender porque dicho comportamiento y los precios de los activos se desvían de las prescripciones del modelo.

Zurita (2005), coincide en que la tasa de descuento utilizada en la valoración de activos, es de vital importancia al momento de aplicar criterios de inversión como el Valor Neto Actual (VAN), y como este podría perder su validez, si la determinación de la mencionada tasa de descuento no es la correcta. Se incorpora una nueva variable, y es la diferencia que ocurre al calcular una tasa de descuento cuando los proyectos a valorar corresponden ya sea al sector privado o al sector social. Cuando existe esta diferencia, varios factores de riesgo o rendimiento entrarían en discusión, dejando en ciertos casos la normativa positiva del VAN sin efecto.

Un método alternativo para valorar proyectos, especialmente en mercados emergentes, podría complementarse con lo planteado por Sabal (2002) ajustando los flujos futuros del proyecto, mediante un modelo que permita predecir el tipo de cambio efectivo basado en experiencias previas, y dependiendo de la factibilidad de esta predicción. En caso de que el riesgo de tipo de cambio no pueda ser estimado, y este no se pueda diversificar, se debería agregar a la tasa de descuento, una prima de riesgo que el inversor está asumiendo por invertir en un país ajeno al de su origen (Hamard y Lamothe, 2009). 
Estos autores mencionan que, de acuerdo con evidencias empíricas, el riesgo de tipo de cambio es muy volátil en países de América Latina, convirtiéndolo a éste en sistemático, además de tener un impacto negativo sobre el rendimiento respectivo (Dos Santos, Klotzle, y Pinto, 2021). Esto último se debe en parte a la tendencia histórica de la devaluación de las monedas y las crisis económicas que se han originado en los países de mercados emergentes (Timurlenk y Kaptan, 2012). Es por ello que, de acuerdo a Hamard y Lamothe (2009) lo adecuado sería hacer un ajuste respectivo a la tasa de descuento para descontar los flujos futuros a valor presente.

Vélez-Pareja y Tham (2010) señala que la valoración de activos o proyectos no depende únicamente de la correcta proyección o estimación de flujos futuros, sino también en la adecuada estructura de capital de la empresa, ya sea por capital común, preferente o mediante deuda puesto que la misma tendría incidencia directa en la valoración de proyectos. Warnes y Warnes (2014) indican que los modelos de CAPM que toman en cuenta mecanismos de ajuste del riesgo país, son válidos cuando existen mercados completamente integrados, además de no existir evidencia teórica o empírica, que el costo de capital de cada empresa, indistintamente de su industria, conlleve una correlación estrecha con el riesgo país. De acuerdo con Fernández (2016) el modelo CAPM tiene muchos errores, puesto que este modelo basa sus hipótesis y conclusiones en escenarios que no son reales. Una de las particularidades que es mayormente cuestionada por Fernández (2014) es que el CAPM asume que los inversionistas tienen expectativas homogéneas, tanto en riesgo como en rendimiento, además de asumir que la composición de los instrumentos de renta variable de un portafolio es similar en todos los inversionistas. Otra de las suposiciones del modelo CAPM, es que se puede invertir y adquirir un préstamo a la misma tasa libre de riesgo, lo cual no es real, puesto que existen diferentes volatilidades en los betas, cada uno identificado con la industria a la que pertenece (Fernández, 2014).

El cálculo de los betas es incorrecto, específicamente si estos se derivan de datos históricos (Fernández y Carabias, 2007). La explicación propuesta radica en varios factores muy simples, y es que estos indicadores de riesgo cambian constantemente, casi a diario, y dependen de qué índice bursátil se tomó como referencia (Fernández, 2016). De igual manera se menciona que estos indicadores están sujetos al referencial de tiempo tomado, y de las rentabilidades empleadas para su cálculo, exponiendo la poca relación que existe en el desempeño entre empresas, y la correlación de las regresiones utilizadas para el cálculo no son significativas. El modelo CAPM erróneamente asume que la rentabilidad exigida a las acciones debería ser la misma para todos los inversionistas (Fernández, 2008), puesto que todos deberían aplicar el beta y prima de riesgo del mercado. Los flujos de caja que se estiman para la valoración, no son los mismos para todos los inversionistas, ya que estos se basan en distintas 
expectativas de las industrias y percepción de la economía (Fernández, 2011). Es decir, es aceptable tener diferentes expectativas de flujos de caja entre profesionales, pero no se admite distintas apreciaciones del riesgo. El modelo CAPM combina la relación entre riesgo y rendimiento, pero no se puede hablar de un beta para todo el mercado, ya que cada inversor tiene diferente exposición al riesgo, diferente apreciación de la realidad económica y por ende diferente beta y rendimiento del mercado, factores utilizados al momento de calcular el CAPM.

Por otra parte, la valoración de inversiones, instrumentos financieros, empresas y proyectos, dependen en gran medida del tipo de cambio de moneda, el cual de acuerdo con Kozikowski (2013) se refiere al precio de una moneda extranjera en términos de la moneda local. El tipo de cambio o el riesgo de tipo de cambio, es una variable que ciertos países tienden a manipular, ya sea a través de la devaluación de la moneda provocada por el banco central con el fin de ganar competitividad en las exportaciones, fomentar el turismo e incentivar el consumo de bienes y servicios locales. Este riesgo en mención no es tomado en cuenta o valorado acertadamente para el cálculo de una tasa descuento, especialmente en países de la región Andina (Qi, 2010).

Inversionistas, especialmente provenientes de economías desarrolladas, han auscultado oportunidades de diversificación mediante Inversiones Extranjeras Directas (IED) especialmente en mercados emergentes (Roggi, Giannozzi, y Baglioni, 2017). Esta tendencia, sin embargo, no se ha replicado completamente en los mercados emergentes de América Latina, debido a que en estos países se evidencia mayor inestabilidad política y volatilidad que afectan los indicadores macroeconómicos (Martínez, Ledesma, y Russo, 2013). Lo señalado, se puede considerar como un ejemplo, en donde la falta de información relevante y oportuna acerca de factores de medición de riesgo y rentabilidad, además de subjetividad para adjudicar los mismos, generan incertidumbre en los inversionistas, reduciendo así las inversiones extranjeras directas.

Para una mejor comprensión de lo expuesto, la Tabla 1 muestra un resumen de las ideas más importantes sobre el CAPM y su aplicación en mercados emergentes.

\section{Metodología}

La metodología utilizada para la investigación se basa en una revisión descriptiva y analítica de la teoría publicada desde el año 1964 hasta el año 2021. En primer lugar mediante un proceso de selección de los artículos seminales, que son aquellos publicados por los mayores exponentes sobre el modelo de valoración de activos financieros CAPM. En un segundo momento, se obtuvieron datos sobre los artículos en base de datos Scopus, en la biblioteca digital JSTOR, en la red Redalyc y el sistema Dialnet utilizando como términos clave para las búsquedas 
Tabla 1.

Principales aportes al CAPM y su aplicación en mercados emergentes

\begin{tabular}{|c|c|c|}
\hline Autor & Principio & Conclusión \\
\hline $\begin{array}{l}\text { Markowitz } \\
(1952)\end{array}$ & $\begin{array}{l}\text { Plantea un Modelo de eficiencia de Portafolio } \\
\text { donde se analiza la relación existente entre el } \\
\text { riesgo y rendimiento. }\end{array}$ & $\begin{array}{l}\text { Al juntar varios instrumentos financieros en una cartera, } \\
\text { se puede optimizar el rendimiento y el riesgo respectivo } \\
\text { tomando en cuenta la ponderación de los mismos en un } \\
\text { portafolio y su debida correlación. }\end{array}$ \\
\hline $\begin{array}{l}\text { Sharpe } \\
(1964)\end{array}$ & $\begin{array}{l}\text { Introduce el "Modelo de Mercado" el cual señala } \\
\text { la relación directa que existe entre el riesgo y el } \\
\text { retorno esperado de una inversión, pero tomando } \\
\text { en cuenta los riesgos sistemáticos y no } \\
\text { sistemáticos, siendo la suma de estos dos, el riesgo } \\
\text { total del portafolio. }\end{array}$ & $\begin{array}{l}\text { Ya que el riesgo sistemático no es diversificable, un } \\
\text { portafolio bien diversificado, eliminaría el riesgo No } \\
\text { Sistemático, así contemplando expresamente el riesgo } \\
\text { sistemático. }\end{array}$ \\
\hline $\begin{array}{l}\text { Sharpe } \\
(1964)\end{array}$ & $\begin{array}{l}\text { "Modelo de Valoración de Activos Financieros } \\
\text { CAPM" (Capital Asset Pricing Management). Se } \\
\text { introduce para este modelo un indicador de la } \\
\text { volatilidad de un activo con relación al mercado } \\
\text { llamado "beta". Este modelo asume, que el } \\
\text { mercado es perfectamente competitivo, no existe } \\
\text { asimetría de información. }\end{array}$ & $\begin{array}{l}\text { Este modelo sirve para valo rar tanto instrumentos } \\
\text { financieros como para valorar proyectos. El modelo } \\
\text { CAPM, por el efecto de la diversificación, no presenta } \\
\text { riesgo No Sistemático, solo sistemático, planteando una } \\
\text { relación creciente entre beta, rendimiento y riesgo. }\end{array}$ \\
\hline $\begin{array}{l}\text { Merton } \\
(1973)\end{array}$ & $\begin{array}{l}\text { "Modelo CAPM Multifactor" existen varios } \\
\text { riesgos que afectan el poder adquisitivo o recursos } \\
\text { del inversionista aparte de la incertidumbre de } \\
\text { precio. }\end{array}$ & $\begin{array}{l}\text { Este modelo se enfoca en la prima de riesgo, y manifiesta } \\
\text { acerca de la compensación que los inversionistas } \\
\text { deberían por cada riesgo adicional asumido además del } \\
\text { mercado. }\end{array}$ \\
\hline Ross (1976) & $\begin{array}{l}\text { "Modelo de la Teoría por Valoración de Arbitraje } \\
\text { o APT" (Arbitraje Pricing Theory ) que tiene como } \\
\text { objetivo, estimar la prima de riesgo, y su } \\
\text { rentabilidad condicionada por factores de carácter } \\
\text { anticipables y no anticipables. }\end{array}$ & $\begin{array}{l}\text { El modelo APT es menos restrictivo o más flexible en } \\
\text { cuanto a la preferencia de los inversionistas con relación } \\
\text { al riesgo y al rendimiento. }\end{array}$ \\
\hline $\begin{array}{l}\text { Breeden } \\
(1979)\end{array}$ & $\begin{array}{l}\text { Propone el C-CAPM para medir el rendimiento } \\
\text { incorporando al consumo. }\end{array}$ & $\begin{array}{l}\text { Los datos reales de consumo pueden tener errores en su } \\
\text { medición, al contrario que el precio de las acciones. }\end{array}$ \\
\hline $\begin{array}{l}\text { Mariscal \& } \\
\text { Lee }(1993)\end{array}$ & $\begin{array}{l}\text { Proponen la modificación del Modelo CAPM por } \\
\text { la adición del indicador Riesgo País. }\end{array}$ & $\begin{array}{l}\text { Se procura compensar la exposición al riesgo adicional } \\
\text { que inversionistas en países con mercados desarrollados } \\
\text { asumen al invertir en mercados emergentes. }\end{array}$ \\
\hline $\begin{array}{l}\text { Estrada } \\
(2000)\end{array}$ & $\begin{array}{l}\text { Propone el D-CAPM utilizando para este cálculo } \\
\text { el beta a la baja. }\end{array}$ & $\begin{array}{l}\text { Brinda mejores resultados al ser aplicado en mercados } \\
\text { emergentes. }\end{array}$ \\
\hline $\begin{array}{l}\text { Sabal } \\
(2002)\end{array}$ & $\begin{array}{l}\text { El Riesgo País no debería ser incluido en el } \\
\text { modelo CAPM puesto que esto implicaría que este } \\
\text { riesgo es Sistemático. Plantea un mecanismo de } \\
\text { ponderación de los betas de las industrias que } \\
\text { conforman los mercados en vías de desarrollo }\end{array}$ & $\begin{array}{l}\text { El riesgo país ya estaría incluido al calcular la prima de } \\
\text { riesgo usando los bonos soberanos del país en estudio, } \\
\text { como la tasa libre de rie sgo, especialmente en economías } \\
\text { emergentes. Con el mecanismo de ponderación de betas } \\
\text { se podría valorar activos financieros de manera más } \\
\text { precisa. }\end{array}$ \\
\hline $\begin{array}{l}\text { Damodaran } \\
(2002)\end{array}$ & $\begin{array}{l}\text { Sugiere que al riesgo país, se le debería ajustar la } \\
\text { desviación de la prima de riesgo de un mercado } \\
\text { accionario emergente, con la prima de riesgo de un } \\
\text { mercado desarrollado además de la inclusión de un } \\
\text { factor llamado Lambda a la ecuación. }\end{array}$ & $\begin{array}{l}\text { Debido al ajuste de la relación riesgo y rentabilidad en } \\
\text { mercados de economías emergentes, y la ponderación } \\
\text { del riesgo país a diferentes industrias y proyectos con la } \\
\text { inclusión del factor Lambda, se lograría valor } \\
\text { instrumentos financieros de manera más acertada. }\end{array}$ \\
\hline $\begin{array}{l}\text { Fernández } \\
(2011- \\
2016)\end{array}$ & $\begin{array}{l}\text { El modelo CAPM es incorrecto puesto a que éste } \\
\text { basa sus hipótesis y conclusiones en escenarios } \\
\text { que no son reales además de depender del índice } \\
\text { bursátil que se tomó como referencia, puesto que } \\
\text { estos cambian constantemente. }\end{array}$ & $\begin{array}{l}\text { El modelo CAPM incorrectamente asume que la } \\
\text { rentabilidad exigida a las acciones debería ser la misma } \\
\text { para todos los inversionistas .Los flujos de caja que se } \\
\text { estiman para la valoración, no son los mismos para todos } \\
\text { los inversionistas, ya que estos se basan en distintas } \\
\text { expectativas de las industrias y percepción de la } \\
\text { economía. }\end{array}$ \\
\hline
\end{tabular}

Fuente: Elaboración propia. 
"capm" y "mercados emergentes" dando como resultado un total de 9028 artículos у 659 artículos respectivamente. La etapa de selección de documentos se realizó sin discriminar trabajos tanto en español como en inglés, escogiendo de manera sistemática y cronológica las publicaciones más relevantes en función del número de citas que se tenía de cada artículo. Con todas estas consideraciones se seleccionaron inicialmente 50 trabajos pero durante el proceso de fichaje de cada documento, se eliminaron aquellos que no poseían información relevante para el estudio sustituyendo estos documentos con otros presente es la base de datos. llegando a utilizar finalmente 54 documentos que constan en las referencias finales.

\section{Resultados}

Los modelos de valoración que generalmente se utilizan, si bien pueden ser alineados y relevantes para economías desarrolladas o en mercados emergentes, no reflejan en sí, un mecanismo óptimo para la valoración de proyectos en países cuyos mercados de valores son realmente deficientes (Mercados Sub-Emergentes de Latinoamérica), y sus variables macroeconómicas mencionadas en este ensayo, difieren significativamente, conllevando a conclusiones con mayor grado de subjetividad o incertidumbre.

Por lo señalado a través del estado del arte, este trabajo de revisión literaria presenta que al no contar con una tasa de descuento apropiada para valorar proyectos o instrumentos financieros en países de economías sub-emergentes y la no existencia de una metodología adecuada para estos procesos, se expone la problemática de la sobrevaloración o subvaloración de los distintos proyectos o instrumentos financieros. Es claro que es necesario ajustar el modelo CAPM como mecanismo de valoración no solo hacia un país o región, sino que también hacia la realidad de cada industria, empresa o proyecto de manera de reducir disparidades en cuanto a variables macroeconómicas, y así obtener resultados, si bien no perfectos, pero lo suficientemente óptimos que nos acerquen hacia la correcta toma de decisiones.

\section{Conclusiones}

El modelo CAPM ha sido el más popular para el efecto desde su aparición en 1964. Esta popularidad se debe a la relación que representa entre el rendimiento de un activo y su riesgo. Sin embargo, su sustento teórico se basa en economías donde los mercados tienen ciertas condiciones que no pueden aplicarse en todas partes. Debido a esto, el modelo ha experimentado varias adaptaciones a través de los años con el fin de que se lo pueda utilizar en otros tipos de mercados como los emergentes. La literatura evidencia que al no existir una tasa de descuento apropiada para valorar proyectos o instrumentos financieros en países de economías emergentes y la no existencia de una metodología adecuada para estos procesos, se expone la problemática de la sobrevaloración o subvaloración de los mismos. Es claro que la mayoría de propuestas para adaptar el modelo CAPM a las economías emergentes han utilizado la ecuación base desarrollada originalmente por Sharpe. 
Para una importante cantidad de autores, la única variable que explica la rentabilidad es el coeficiente beta. En mercados emergentes existen otros factores que deben considerarse para entender el verdadero riesgo al que se exponen los inversionistas en estos mercados. Las economías emergentes también resultan complejas de clasificar, existen países, considerados en vías de desarrollo, con altos niveles de ingresos, reflejados en su PIB, que pueden superar incluso el ingreso de países que se consideran desarrollados. Además, de las diferencias de ingresos, dentro de los países emergentes existen otras variables que dificultan la valoración de los proyectos, como es el caso de la política monetaria y el riesgo país. A pesar de todo esto, los mercados emergentes atraen a una gran cantidad de inversionistas extranjeros, debido principalmente a que sus índices de crecimiento son superiores a los de las economías desarrolladas y sus poblaciones concentran a una gran cantidad de personas que resultan ideales para la apertura de nuevas inversiones. En este estudio se puso de manifiesto que existen varias fórmulas con diferentes variables propuestas por autores que han tratado de acomodar el modelo original CAPM a las condiciones que presentan los mercados emergentes. Sin embargo, queda claro que no existe una fórmula universal.

\section{Referencias}

Abuaf, N. (2011). Valuing Emerging Market Equities-The Empirical Evidence. Journal of Applied Finance, 2, 1-19. https://ssrn.com/abstract=2691604
Álvarez, R., Ortega, G., Sánchez, A., y Herrera, M. (2004). Evolución de la teoría económica de las finanzas: una breve revisión. Semestre Económico, 7(14), 105-127. http://www.redalyc.org/articulo.oa?id=16 5013658004

Bai, Y., y Green, C. (2020). Country and industry factors in tests of Capital Asset Pricing Models for partially integrated emerging markets. Economic Modelling, 92, 180-194. https://doi.org/10.1016/j.econ $\bmod .2019 .12 .019$

Bekaert, G., y Harvey, C. R. (2013). Emerging Equity Markets in a Globalizing World. SSRN Electronic Journal. https:// doi.org/10.2139/ssrn.2344817

Botero, D., y Vecino, C. (2015). Modelación de la relación rentabilidad-riesgo en el mercado accionario para países desarrollados y países emergentes en un mundo parcialmente integrado. Cuadernos de Administración, 31(53), 38-47. https://www.redalyc.org/articulo. oa? id=225040779004

Breeden, D. (1979). An intertemporal asset pricing model with stochastic consumption and investment opportunities. Journal of Financial Economics, 7(3), 265-296. https://doi.org/https://doi.org/10.1016/03 04-405X(79)90016-3

Britzelmaier, B. (2009). Company Valuation in Emerging Markets. International Journal of Management Cases, 12(2), 750-759. https://doi.org/10.5848/APBJ.2010.00112

Bruner, R., Conroy, R., Estrada, J., Kritzman, M., y Li, W. (2002). Introduction to "valuation in emerging markets." Emerging Markets Review, 3(4), 310-324. https://doi.org/10.1016/S15660141(02)00039-0

Butt, H., y Sadaqat, M. (2020). The pricing of firm-specific risk in emerging markets Specification errors of Asset pricing 
models View project Liquidity and asset pricing View project. Journal of Risk, 8(4), 21-32. https://doi.org/10.21314/JO IS. 2020.115

Campos, S., Castro, M., Cuy, M., y Ferrer, G. (2005). CAPM en mercados emergentes. In Universitat Pompeu Fabra. Universitat Pompeu Fabra. https://www.bsm.upf.edu/ documents/mmf/04_01_capm.pdf

Cavusgil, S. (2021). Advancing knowledge on emerging markets: Past and future research in perspective. International Business Review, 30(2), 101796. https:// doi.org/10.1016/j.ibusrev.2021.101796

Damodaran, A. (2002). Investment valuation: Tools and techniques for determining the value of any asset No Title (J. W. and Sons (ed.); 2nd ed. John Wiley and Sons.

Damodaran, A. (2003). Measuring company exposure to country risk: theory and practice. SSRN Electronic Journal, September, 30. https://doi.org/http://dx. doi.org/10.2139/ssrn. 889388

Damodaran, A. (2007). Valuation approaches and metrics: A survey of the theory and evidence. Foundations and Trends in Finance, 1(8), 693-784. https://doi.org/ $10.1561 / 0500000013$

Dos Santos, M. Klotzle, M., y Pinto, A. (2021). The impact of political risk on the currencies of emerging markets. Research in International Business and Finance, 56, 101375. https://doi.org/10.1016/ j.ribaf.2020.101375

Elbannan, M. (2015). The Capital Asset Pricing Model: An Overview of the Theory. International Journal of Economics and Finance, 7(1), 216-228. https://doi.org/ 10.5539/ijef.v7n1p216

Estrada, J. (2002). Systematic risk in emerging markets: The D-CAPM. Emerging Markets Review, 3(4), 365-379. https://
doi.org/10.1016/S1566-0141(02)00042-0

Fama, E., y French, K. (1996). The CAPM is wanted, dead or alive. Journal of Finance, 51(5), 1947-1958. https://doi. org/10.1111/j.1540-6261.1996.tb05233.x

Fama, E., y French, K. (2003). The CAPM: Theory and Evidence. Journal of Economic Perspectives, 18(03), 25-46. https:// doi.org/10.1257/0895330042162430

Fama, E., y French, K. (2004). The Capital Asset Pricing Model: Theory and Evidence. Journal of Economic Perspectives, 18(3), 25-46. https://doi.org/10.1257/08953300 42162430

Fernández, P. (2008). Valoración de empresas por descuento de flujos: diez métodos y siete teorías. In Documento de Investigación (D/766; IESE Research Papers, Vol. 3). https://ideas.repec.org/p/ebg/iesewp/d-07 66.html

Fernández, P. (2011). Discounted Cash Flow Valuation Methods: Examples of Perpetuities, Constant Growth and General Case. SSRN Electronic Journal, 1-25. https://doi.org/10.2139/ssrn.743229

Fernández, P. (2014). CAPM (capital asset pricing model): un modelo absurdo. SSRN Electronic Journal, 1-16. https:// doi.org/ http://dx.doi.org/10.2139/ssrn.2499455

Fernández, P. (2016). Métodos de valoración de empresas. SSRN Electronic Journal, 1-28. https://doi.org/http://dx.doi.org/10. 2139/ssrn. 1267987

Fernández, P., y Carabias, J. (2007). El Peligro de utilizar Betas Calculadas. SSRN Electronic Journal, 3, Article D/685. https://doi.org/10.2139/ssrn.897700

García-Sánchez, J., Preve, L., y Sarria-Allende, V. (2010). Valuation in Emerging Markets: A Simulation Approach. Journal of Applied Corporate Finance, 22(2), 100-108. 
https://doi.org/10.1111/j.1745-6622.2010 $.00279 . x$

Godfrey, S., y Espinosa, R. (1996). A practical approach to calculating costs of equity for investments in emerging markets. Journal of Applied Corporate Finance, 9(3), 80-90. https://doi.org/10.1111/j.1745-662 2.1996.tb00300.x

Hamard, A., y Lamothe, P. (2009). A modified CAPM Valuation Model for Latin American Emerging Markets. Documentos de Trabajo En Finanzas de Empresas, 1, 1-26. https://dialnet.uni rioja.es/servlet/articulo? codigo $=2957154$

Harvey, C. (2005). Country Risk Components, the Cost of Capital, and Returns in Emerging Markets. SSRN Electronic Journal. https://doi.org/10.2139/ssrn.620710

Kiguel, M., y Lopetegui, G. (1997). Entendiendo el Riesgo País. In CEMA Working Papers: Serie Documentos de Trabajo (No. 125). https://www.ucema.edu.ar/pu blicaciones/download/documentos/125.pdf

Kozikowski, Z. (2013). Finanzas Internacionales (3rd ed.). McGraw-Hill Companies.

Kruschwitz, L., Löffler, A., \& Mandl, G. (2012). Damodaran's Country Risk Premium: A Serious Critique. Business Valuation Review, 31(2-3), 1-20. https://doi.org/ http://dx.doi.org/10.2139/ssrn.1651466

Lee, C., y Wang, E. (2020). Economic Complexity and Income Inequality: Does Country Risk Matter? Social Indicators Research, 154(1), 35-60. https://doi.org/10.1007/ s11205-020-02543-0

Mariscal, J., y Lee, R. (1993). The valuation of Mexican stocks: an extension of the capital asset pricing model to emerging markets. In Goldman Sachs Investment Research (Goldman Sachs Investment Research). https://faculty.fuqua.duke. edu/ charvey/Teaching/BA456_2006/GS
_The_valuation_of_mexican_stocks.pdf

Markowitz, H. (1952). Portfolio selection. The Journal of Finance, 77(1), 77-91. https://doi.org/https://doi.org/10.2307/29 75974

Martínez, C., Ledesma, J., y Russo, A. (2013). Particularidades del Modelo de Fijación de Precios de Activos de Capital (CAPM) en Mercados Emergentes. Análisis Financiero, 121, 37-47. https://dialnet. unirioja.es/servlet $/$ articulo? codigo $=4538$ 852

Merton, R. (1973). An Intertemporal Capital Asset Pricing Model. Econometrica: Journal of the Econometric Society, 41(5), 867-887. https://doi.org/https://doi.org/10.2307/19 13811

Obstfeld, M., Ostry, J., \& Qureshi, M. S. (2019). A tie that binds: Revisiting the trilemma in emerging market economies. Review of Economics and Statistics, 101(2), 279-293. https://doi.org/10.1162/rest_a_00740

Pereiro, L. (2006). The practice of investment valuation in emerging markets: Evidence from Argentina. Journal of Multinational Financial Management, 16(2), 160-183. https://doi.org/10.1016/j.mulfin.2005.06.001

Perold, A. (2004). The capital asset pricing model. Journal of Economic Perspectives, 18(3), 3-24. https://doi.org/10.1257/08953300 42162340

Qi, H. (2010). Valuation methodologies and emerging markets. Journal of Business Valuation and Economic Loss Analysis, $5 \quad\left(\begin{array}{c}1 \\ 1\end{array}\right)$ https://doi.org/10.2202/1932-9156.1064

Roggi, O., Giannozzi, A., y Baglioni, T. (2017). Valuing emerging markets companies: New approaches to determine the effective exposure to country risk. Research in International Business and Finance, 39, 553-567. https://doi.org/10. 
1016/j.ribaf.2016.07.028

Ross, S. (1976). The arbitrage theory of capital asset pricing (Working Paper Version). Journal of Economic Theory, 13(3), 341-360. https://doi.org/https://doi.org/ 10.1016/0022-0531(76)90046-6

Sabal, J. (2002). Financial Decisions in Emerging Markets (1st ed.). Oxford University Press.

Sabal, J. (2004). The Discount Rate in Emerging Markets: A Guide. Journal of Applied Corporate Finance, 16(2-3), 155-166. https://doi.org/10.1111/j.1745-6622.2004 .tb00547.x

Sabal, J. (2008). A Practical Approach for Quantifying Country Risk. Journal of Globalization, Competitiveness \& Governability, 2(3), 50-63. https://doi. org/10.3232/GCG.2008.V2.N3.03

Sharpe, W. (1964). Capital Asset Prices: A Theory of Market Equilibrium under conditions of risk. The Journal of Finance, 19(3), 425-442. https://doi.org/10.2307/2977928

Sharpe, W. (1990). Capital Asset Prices with and without Negative Holdings. The Journal of Finance, 46(2), 489-509. https:// doi.org/https://doi.org/10.2307/2328833

Solnik, B. (1974). An equilibrium model of the international capital market. Journal of Economic Theory, 8(4), 500-524. https://doi.org/https://doi.org/10.1016/00 22-0531(74)90024-6

Timurlenk, Ö., y Kaptan, K. (2012). Country Risk. Procedia - Social and Behavioral Sciences, 62, 1089-1094. https://doi.org/ 10.1016/j.sbspro.2012.09.186

United Nations Conference on Trade and Development (UNCTAD). (2020). Informe sobre las inversiones en el mundo 2020. https://unctad.org/ system/ files/officialdocument/wir2020_overview_es.pdf
Vélez-Pareja, I., y Tham, J. (2010). An Introduction to the Cost of Capital. SSRN Electronic Journal, 1-27. https://doi.org/ http:// dx.doi.org/10.2139/ssrn.1570718

Warnes, I., y Warnes, P. (2014). Country risk and the cost of equity in emerging markets. Journal of Multinational Financial Management, 28, 15-27. https://doi.org/ 10.1016/j.mulfin.2014.08.001

Wong, D., y Chirinos, M. (2016). ¿Los modelos basados en el CAPM valoran adecuadamente los emprendimientos familiares? Innnovar, 26(61), 65-82. https://doi.org/10.15446/innovar.v26n61. 57167.G12

Zurita, F. (2005). Un Examen a la tasa de descuento. El Trimestre Económico, 72(286), 257-281. http://www.jstor.org/stable/20856856 
